\title{
Pathophysiological interference with neurovascular coupling - when imaging based on hemoglobin might go blind
}

\author{
Ute Lindauer ${ }^{*}$, Ulrich Dirnagl2,3,4, Martina Füchtemeier ${ }^{2}$, Caroline Böttiger ${ }^{2}$, Nikolas Offenhauser ${ }^{2}$, \\ Christoph Leithner ${ }^{2 \dagger}$ and Georg Royl ${ }^{2 t}$
}

\author{
Department of Neurosurgery, Klinikum rechts der Isar, Technical University, Munich, Germany \\ 2 Departments of Neurology and Experimental Neurology, Charité - Universitätsmedizin Berlin, Berlin, Germany \\ ${ }^{3}$ Center for Stroke Research Berlin, Berlin, Germany \\ ${ }^{4}$ Neurocure Research Center Berlin, Berlin, Germany
}

Edited by:

Anna Devor, University of California San Diego, USA

\section{Reviewed by:}

Tom Liu, University of California San Diego, USA

Kamil Uludag, Max Planck Institute, Germany

Noam Harel, University of Minnesota, USA

\section{*Correspondence:}

Ute Lindauer, Departments of

Neurosurgery, Experimental

Neurosurgery, Klinikum rechts der Isar,

Technical University Munich,

Ismaninger Str. 22, 81675 Munich,

Germany.

e-mail: ute.lindauer@Irz.tum.de

${ }^{+}$Christoph Leithner and Georg Royl contributed equally to the manuscript.
Assessing neuronal activity by non-invasive functional brain imaging techniques which are based on the hemodynamic response depends totally on the physiological cascade of metabolism and blood flow. At present, functional brain imaging with near infrared spectroscopy (NIRS) or BOLD-fMRI is widely used in cognitive neuroscience in healthy subjects where neurovascular coupling and cerebrovascular reactivity can be assumed to be intact. Local activation studies as well as studies investigating functional connectivity between brain regions of the resting brain provide a rapidly increasing body of knowledge on brain function in humans and animals. Furthermore, functional NIRS and MRI techniques are increasingly being used in patients with severe brain diseases and this use might gain more and more importance for establishing their use in the clinical routine. However, more and more experimental evidence shows that changes in baseline physiological parameters, pharmacological interventions, or disease-related vascular changes may significantly alter the normal response of blood flow and blood oxygenation and thus may lead to misinterpretation of neuronal activity. In this article we present examples of recent experimental findings on pathophysiological changes of neurovascular coupling parameters in animals and discuss their potential implications for functional imaging based on hemodynamic signals such as fNIRS or BOLD-fMRI. To enable correct interpretation of neuronal activity by vascular signals, future research needs to deepen our understanding of the basic mechanisms of neurovascular coupling and the specific characteristics of disturbed neurovascular coupling in the diseased brain.

Keywords: BOLD-fMRI, cerebral blood flow, cerebral blood oxygenation, cortical spreading depression, deoxy-hemoglobin, functional activation, hemodynamic response, neurovascular coupling

\section{PHYSIOLOGY OF THE HEMODYNAMIC RESPONSE DURING INCREASED NEURONAL ACTIVITY}

In the brain, coupling of neuronal activity and metabolism with regional cerebral blood flow (rCBF), and cerebral blood oxygenation (rCBO) is tight ("neurovascular coupling"), so rCBF or $\mathrm{rCBO}$ changes can be used to map brain activity with high spatial resolution ("functional neuroimaging"). Considerable progress has been made over the past decades in understanding the physiological mechanisms of neurovascular coupling, but key mechanisms underlying the regulation of brain blood flow and metabolism remain elusive. Tissue factors such as $\mathrm{K}^{+}, \mathrm{H}^{+}, \mathrm{NO}$, or adenosine (Iadecola, 2004) have been center stage ever since 1890, when Roy and Sherrington (1890) first proposed that rCBF is regulated by local metabolites. It has long been suggested that the increase in CBF during neural activation is driven by a need for increased delivery of oxygen or glucose. During functional activation, oxygen metabolism (cerebral metabolic rate of oxygen, $\mathrm{CMRO}_{2}$ ) increases substantially (Ances et al., 2001b; Gjedde et al., 2002; Dunn et al., 2005), and a regional and transient change in tissue oxygenation occurs. Increased oxygen consumption during neuronal activity results in a decrease in tissue oxygenation which is counteracted by the increase in $\mathrm{O}_{2}$ supply when $\mathrm{CBF}$ increases (Ances et al., 2001a; Thompson et al., 2003; Offenhauser et al., 2005; Enager et al., 2009).

Oxidative metabolism and CBF exert not only counteracting effects on tissue oxygenation but also on hemoglobin oxygenation. On one hand, increased oxygen metabolism drives conversion of oxygenated (oxy- $\mathrm{Hb})$ to deoxygenated hemoglobin (deoxy-Hb). On the other hand, a disproportionately large increase in regional $\mathrm{CBF}$ leads to a washout of deoxy- $\mathrm{Hb}$ from the activation area, resulting in a decrease of deoxy- $\mathrm{Hb}$ and an increase of oxy- $\mathrm{Hb}$ (Obrig et al., 1996; Lindauer et al., 2001). CBF increases exceed $\mathrm{CMRO}_{2}$ increases by a factor of 2-10 (Fox et al., 1988; Lin et al., 2008 and references therein). The exact value is still intensely debated. Methodological issues regarding $\mathrm{CMRO}_{2}$ measurement might play a relevant role, especially in fMRI (Lin et al., 2008) and the factor likely varies with stimulation parameters and between brain regions and species. However, consensus exists that the increase in CBF is mostly accompanied by tissue hyperoxygenation. The washout and decrease of deoxy- $\mathrm{Hb}$ provides the basis for functional neuroimaging with BOLD-fMRI. The relation of metabolic and vascular events during functional activation is illustrated in Figure 1. 


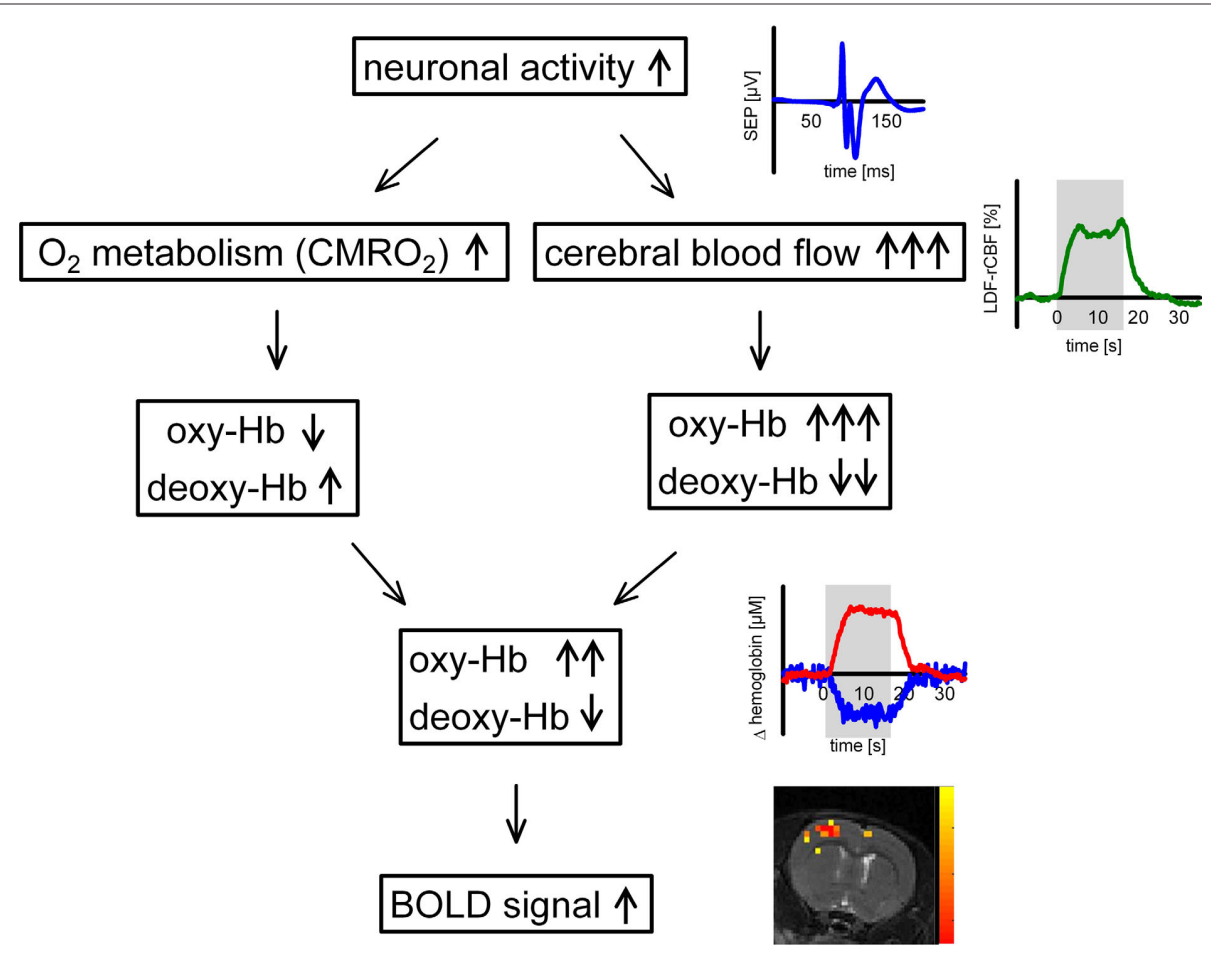

FIGURE 1 | Physiology of the hemodynamic response during increased neuronal activity.

In BOLD-fMRI, the signal transduction of neuronal activity is even more complex and influenced by methodological aspects (e.g., spin echo vs. gradient echo sequences) and field strength (Uludag et al., 2009). However, a close relation between the fMRI-BOLD signal and the deoxy-Hb signal measured with optical imaging has been demonstrated repeatedly, e.g., in a recent study with concurrent measurements by Kennerley et al. (2009). Furthermore, a close correlation has been shown recently between fMRI signal changes and synaptic activity, whereas the correlation with cellular action potentials appears to be weaker (Mathiesen et al., 1998, 2000; Logothetis et al., 2001; Rauch et al., 2008). These findings are congruent with the very recent finding that action potentials are highly energy-efficient, which means that their contribution to activity-dependent metabolism is minimal (Alle et al., 2009).

As described, assessing neuronal activity by non-invasive functional brain imaging techniques which are based on the hemodynamic response depends totally on the physiological cascade of metabolism and blood flow. At present, functional brain imaging with near infrared spectroscopy (fNIRS) or by BOLD-fMRI is widely used in cognitive neuroscience in healthy subjects where neurovascular coupling and cerebrovascular reactivity can be assumed to be intact. Local activation studies as well as studies investigating functional connectivity between brain regions of the resting brain provide a rapidly increasing body of knowledge on brain function in humans and animals (for recent reviews see Obrig and Villringer, 2003; Auer, 2008; Logothetis, 2008; He and Raichle, 2009; Lu et al., 2010). However, more and more experimental evidence shows that changes in baseline physiological parameters, pharmacological interventions, or disease-related vascular changes may significantly alter the normal response of blood flow and blood oxygenation in amplitude as well as time course dynamics and thus may lead to misinterpretation of neuronal activity. Functional MRI techniques are increasingly being used in patients with severe brain diseases, even in patients with vegetative or minimally conscious state (Monti et al., 2010) and this use might increase substantially in the future. To exactly understand the effect of pathophysiology on the mechanisms of neurovascular coupling we need systematic basic research on these interferences. Animal studies on neurovascular coupling are different from human functional activation studies in many aspects. Among others, anesthesia, surgery, small brain size, lack of gyration in rodent brain, and different functional activation systems produce uncertainties when translating the results to the human brain. However, despite this limitation, animal studies deliver important complementary information on blood flow and blood oxygenation changes for human NIRS and fMRI since they allow invasive procedures and multimodal imaging with high signal-to-noise ratio to systematically investigate these pathophysiological influences.

In the following we will therefore summarize examples of recent experimental findings on pathophysiological changes of neurovascular coupling parameters in animals from our group and from others and discuss potential implications for functional imaging based on hemodynamic signals such as NIRS and BOLD-fMRI. 


\section{EXAMPLES OF RECENT EXPERIMENTAL FINDINGS ALTERED BASELINE CONDITIONS: EFFECTS OF CHANGES IN BASELINE BLOOD FLOW, BLOOD OXYGENATION, BODY TEMPERATURE, AND INTRACRANIAL PRESSURE ON HEMODYNAMIC SIGNALS}

In studies mapping brain activity, baseline blood flow and baseline blood oxygenation may differ between subjects and between sessions within the same subject. In addition, factors like premedication or anxiety may influence the baseline conditions in patients. Several studies therefore investigated whether baseline cerebral perfusion and oxygenation significantly influence activation induced deoxy-Hb changes and thus the BOLD signal. When modeling the hemodynamic response to brain activation Buxton et al. (2004) predicted a significant reduction of the BOLD response during increased baseline CBF. Their prediction has been confirmed by several experimental studies within the last years. In two recent elegant studies by Jones et al. (2005) and Huppert et al. (2009) using anesthetized animals, hypercapnia was induced to increase baseline perfusion while blood oxygenation and blood flow changes during functional activation were being measured. A perfusion elevation of $\sim 20 \%$ during mild hypercapnia (with no change in neuronal activity or $\mathrm{CMRO}_{2}$ ) resulted in a significantly diminished deoxy- $\mathrm{Hb}$ response, whereas the absolute $\mathrm{CBF}$ (but not the relative) responses remained constant. When baseline perfusion was increased by up to $100 \%$ (stronger hypercapnia), the deoxy-Hb response almost disappeared (Jones et al., 2005). However the notion that $\mathrm{CMRO}_{2}$ and neuronal activity do not change during hypercapnia has recently been questioned (Zappe et al., 2008) and is still matter of a controversial debate (Chen and Pike, 2010).

As long as neuronal activity and $\mathrm{CMRO}_{2}$ remain constant, an increase in baseline blood flow results in blood hyperoxygenation. In our own studies, we therefore addressed the effect of manipulations of baseline blood oxygenation on deoxy-Hb responses. During hyperoxia the deoxy-Hb outwash was significantly reduced under normobaric conditions (Lindauer et al., 2003) or even abolished under hyperbaric conditions of 3 or 4 ATA (Lindauer et al., 2010), while neuronal activity and $\mathrm{CBF}$ responses remained unaltered (Figure 2).

Induced hypothermia is a routine therapeutic intervention in patients after cardiac arrest (Geocadin et al., 2006) and is currently being investigated as a potential neuroprotective treatment in ischemic stroke (Sacco et al., 2007). It is not clear whether fMRI or fNIRS work under these circumstances. In a recent study we have investigated the effect of hypothermia on neurovascular coupling (Royl et al., 2008). Although hypothermia reduced baseline CBF by almost $50 \%$, neurovascular coupling was preserved. Reduction of functional changes in $\mathrm{CBF}$, deoxy- $\mathrm{Hb}$ and $\mathrm{CMRO}_{2}$ followed reductions in neuronal activity during hypothermia (Figure 3 ). Hypothermia therefore seems to be a condition in which functional changes in $\mathrm{rCBF}$ and $\mathrm{rCBO}$ can still provide a reliable correlate of changes in neuronal activity.

It has long been known that ICP has a strong influence on cerebrovascular dynamics (Kety et al., 1948). The influence of ICP on BOLD-fMRI has not been studied so far. Indirect conclusions can be drawn from studies that report an alteration of BOLD-fMRI in patients with an intracranial tumor, which is often accompanied by an increased ICP. Schreiber et al. (2000) have shown that a glial tumor reduced the ipsilateral BOLD-activity, whereas a non-glial tumor did not cause a significant change. In a recent study, we found that the amplitude of the functional deoxy-Hb decrease in rat somatosensory cortex was reduced when ICP was elevated to $7 \mathrm{mmHg}$. At an ICP of $14 \mathrm{mmHg}$ the deoxy-Hb response was close to 0 and even reversed at an ICP of $28 \mathrm{mmHg}$ (Figure 4). Deoxy-Hb amplitude and ICP were significantly correlated $(r=0.64)$. Time matched control animals without ICP elevation did not show relevant changes in stimulus-induced responses of $\mathrm{CBF}, \mathrm{CBV}$, and deoxy-Hb. This data suggests that BOLD-fMRI can be seriously impaired by ICP, e.g., in a patient with a brain tumor and mildly elevated ICP, pre-surgical BOLD-fMRI might not reliably detect functionally eloquent brain areas (Füchtemeier et al., 2010).

\section{ALTERED VASCULAR REACTIVITY: EFFECTS OF REDUCED CBF RESPONSE ON DEOXY-Hb CHANGES DURING FUNCTIONAL ACTIVATION}

During aging or vascular dementia and Alzheimer's disease, impaired vascular reactivity may not only reduce the CBF responses during preserved neuronal activation, but may also significantly alter blood oxygenation changes. In a recently published study, we reduced the CBF responses in anesthetized rats by pharmacologically blocking well-known mechanisms of neurovascular coupling. While the CBF response to functional activation was reduced by approximately $70 \%$, the deoxy-Hb response was abolished (Figure 5) or even reversed into an increase of deoxy-Hb [see Supplementary Figure 3 in Leithner et al. (2010)] instead of the normal outwash response (Leithner et al., 2010). Importantly, COX-2 inhibitors, a common medication in patients, were part of the pharmacological cocktail used to block the CBF response.

Comparable results of inverted BOLD and tissue oxygenation responses were shown by another group using severe systemic hypotension to abolish the CBF response during functional activation (Nagaoka et al., 2006; Masamoto et al., 2008).

To further address this issue, we are currently investigating whether the well-known impairment of vascular reactivity combined with moderate hypoperfusion within the first $2 \mathrm{~h}$ after cortical spreading depression has any impact on blood flow and blood oxygenation responses to somatosensory stimulation in anesthetized rats (Offenhauser et al., 2007). Preliminary and unpublished data from a small pilot study show that within the first $30 \mathrm{~min}$ after CSD, the CBF response to whisker hair deflection in anesthetized rats $(n=5)$ was significantly reduced despite restored neuronal responses. In separate animals we found that under these conditions the deoxy-Hb response is considerably altered $(n=3)$ (Figure 6). Animals were prepared and $\mathrm{CBF}$ and $\mathrm{CBO}$ changes assessed as described in Lindauer et al. (2001).

Increased baseline $\mathrm{CMRO}_{2}$ in parallel with reduced $\mathrm{CBF}$ during resting and stimulated conditions (transcallosal stimulation) after CSD was recently demonstrated by Piilgaard and Lauritzen (2009), and can be considered as a possible cause for the altered deoxy- $\mathrm{Hb}$ response shown in our experiments.

\section{SYNOPSIS OF EXPERIMENTAL DATA}

The examples presented show that whereas some changes in baseline conditions such as hypothermia do not alter neurovascular coupling, other alterations in baseline conditions as well as an impairment of vascular function may have a serious impact 
rCBF response

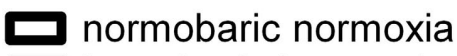

hyperbaric hyperoxia

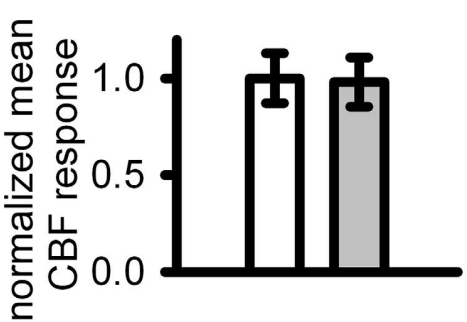

\section{rCBO response}

normobaric hyperbaric

normoxia hyperoxia

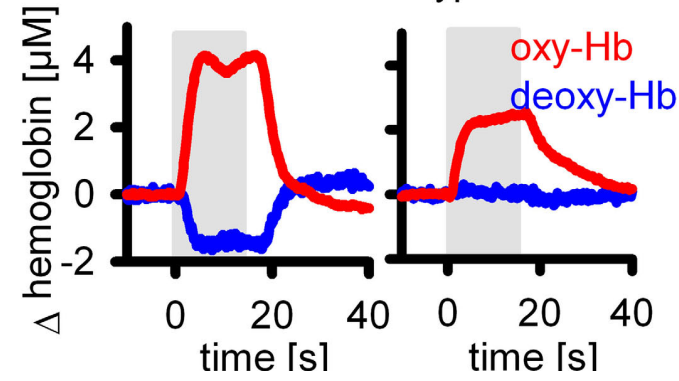

FIGURE 2 | During increased baseline blood oxygenation by hyperbaric hyperoxia, the deoxy-Hb response is abolished (from Lindauer et al., 2010), while the CBF response is unaltered.

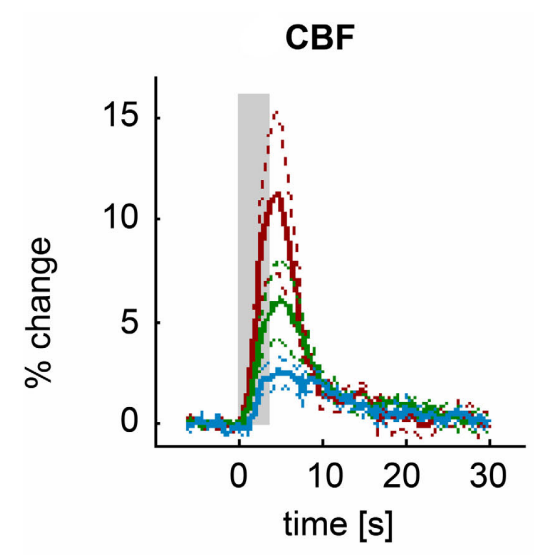

$\mathrm{Hbt}$

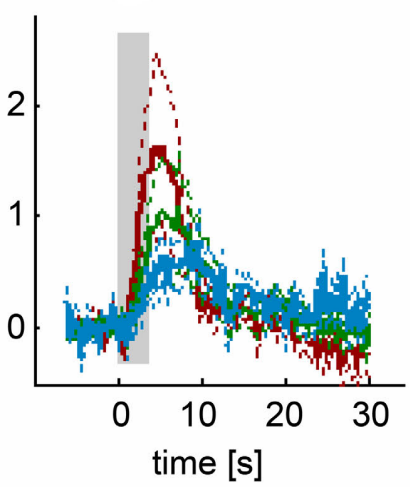

deoxy-Hb

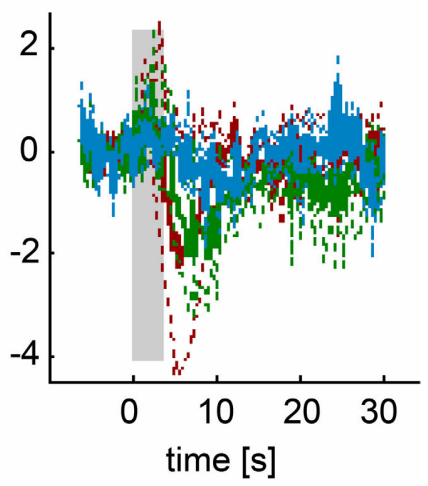

$\mathrm{CMRO}_{2}$

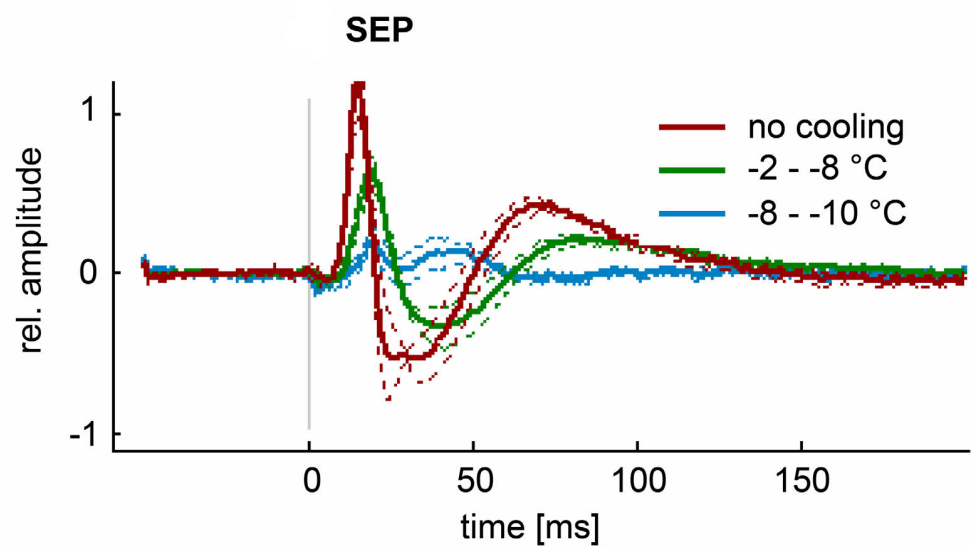

FIGURE 3 | During hypothermia, functional changes in $\mathrm{rCBF}$ and $\mathrm{rCBO}$ (Hbt and deoxy-Hb) still provide a reliable estimate of neuronal activity and oxygen metabolism (SEP and $\mathrm{CMRO}_{2}$ ) (from Royl et al., 2008).

on the hemoglobin derived response. In particular, our finding of significantly changed patterns of deoxy-Hb responses to functional activation during pharmacologically or pathophysiologically reduced $\mathrm{CBF}$ responses clearly points toward the need for a careful interpretation of neuronal function assessed by methods based on vascular parameters like NIRS and BOLD-fMRI whenever vascular reactivity may be impaired. In conclusion, the neurovascular unit can be seriously disturbed by a variety of interferences, and detailed knowledge of the relationship between oxygen metabolism, blood flow, and blood oxygenation will help us to better understand func- 


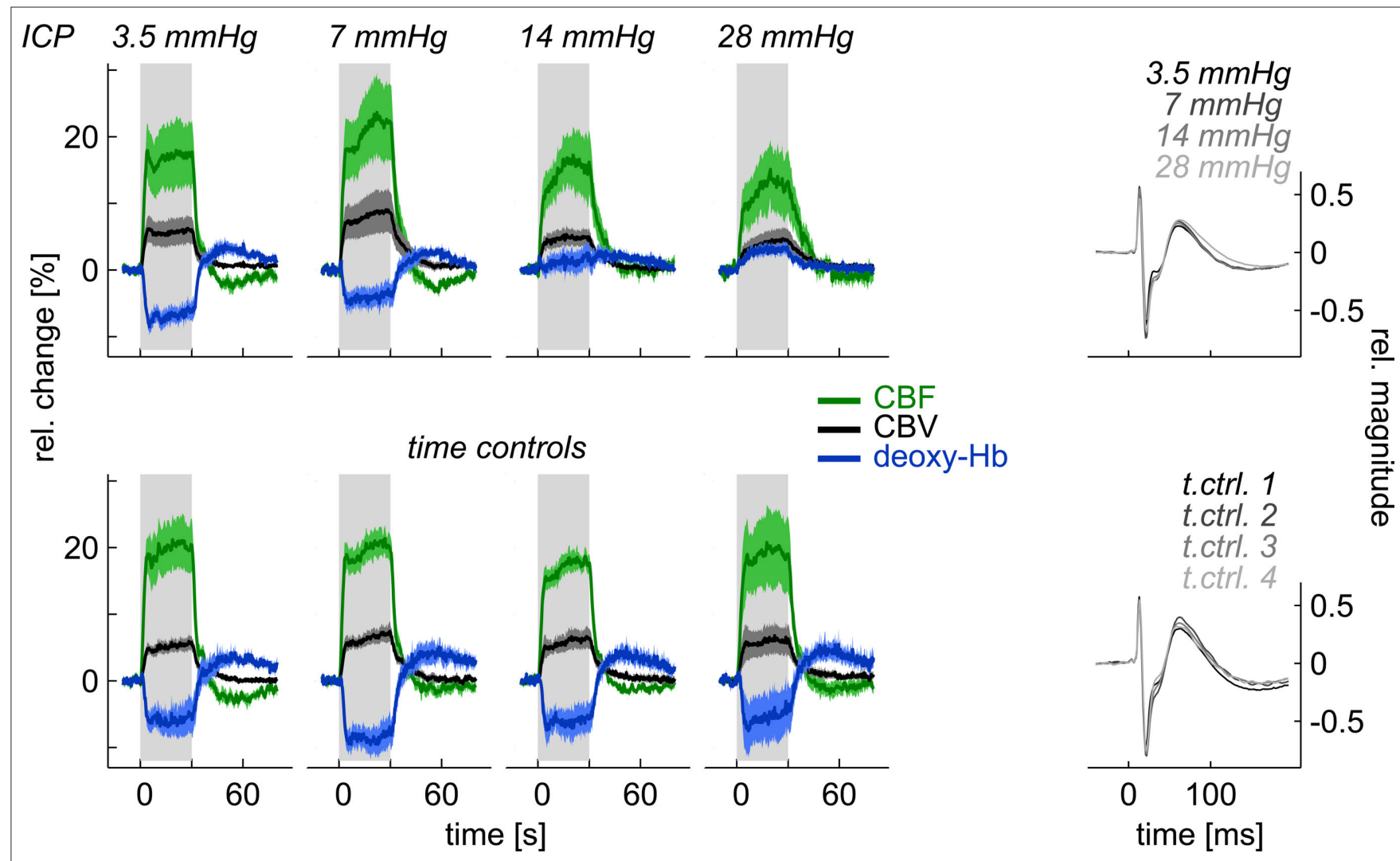

FIGURE 4 |When elevating ICP, functional deoxy-Hb changes can be reduced, leveled out, or reversed, although neuronal activity (SEP) is unchanged and neurovascular coupling persists for CBF and CBV (from Füchtemeier et al., 2010).

tional brain imaging performed on the basis of vascular changes. Further systematic research is needed to achieve this knowledge. Whenever alterations in baseline parameters or vascular reactivity are suspected, it is mandatory that physiological parameters and baseline perfusion be monitored and vascular reactivity tested while using the NIRS or BOLD signal to image brain activation in experimental setups.

\section{POSSIBLE IMPLICATIONS FOR NON-INVASIVE FUNCTIONAL BRAIN IMAGING OF THE DISEASED BRAIN}

Our current knowledge of the complex interaction between metabolic and vascular events during neurovascular coupling leads us to anticipate challenges in the quest for a correct interpretation of data by fNIRS or BOLD-fMRI obtained from the diseased brain. Possible pathophysiological effects on the mechanisms or mediators of neurovascular coupling and/or the impairment of the vascular system's ability to react to released mediators are hitherto largely unknown. Pathophysiological events have the potential to interfere with the signal transduction between neural activity and brain hemoglobin oxygenation (i.e., fNIRS and BOLD-fMRI) at many levels.

In the following paragraphs we will discuss potential implications of these studies. We will specifically focus on BOLD-fMRI because it is the most widely used neuroimaging method and the most likely to be applied in patients due to its non-invasiveness and availability. Furthermore, as implementation of fMRI is more standardized than optical imaging, uncritical application of fMRI in situations of disturbed neurovascular coupling might be somewhat more likely and vulnerability for potential confounders is therefore more important for this technique. Notwithstanding the implications equally apply to optical methods that also play an important role in human studies. Particularly fNIRS is suited for investigating psychiatric patients and children since it does not require as much cooperation.

Several studies have been performed so far to better understand the influence of baseline CBF and of drug effects on functional activation induced NIRS and BOLD signal changes in humans. Beside the use of hypercapnia, the influence of caffeine has been investigated in multimodal approaches. A reduction in baseline $\mathrm{CBF}$ and baseline BOLD signal accompanied by an increase in the evoked CBF and BOLD response (Chen and Parrish, 2009) as well as reduced measures of resting state BOLD connectivity in the motor cortex (Rack-Gomer et al., 2009) have been described after caffeine intake. For indomethacin, a reduction of the baseline BOLD signal and of stimulation induced BOLD and CBF responses during preserved $\mathrm{CMRO}_{2}$ has been reported, whereas acetylsalicylate, another drug regularly used in patients, had no effect on the BOLD signal (Bruhn et al., 2001; St Lawrence et al., 2003). Furthermore, combined approaches of BOLD signal and CBF measurement are gaining importance to achieve a quantitative interpretation of the magnitude of the BOLD effect in relation to oxygen metabolism under normal conditions (Davis et al., 1998; Leontiev and Buxton, 2007) or to investigate whether the coupling relationship between 


\section{baseline}

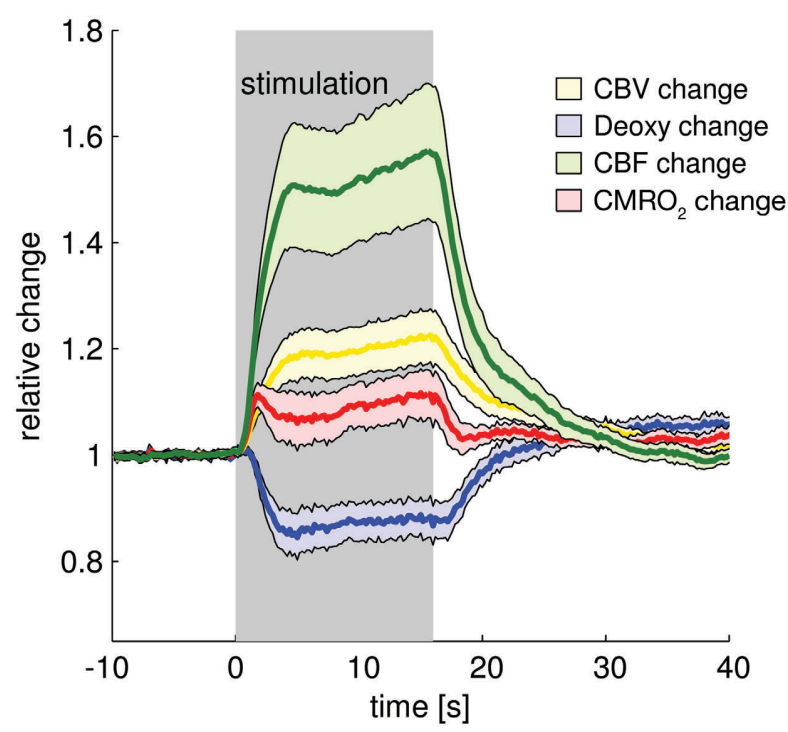

CBF response inhibited

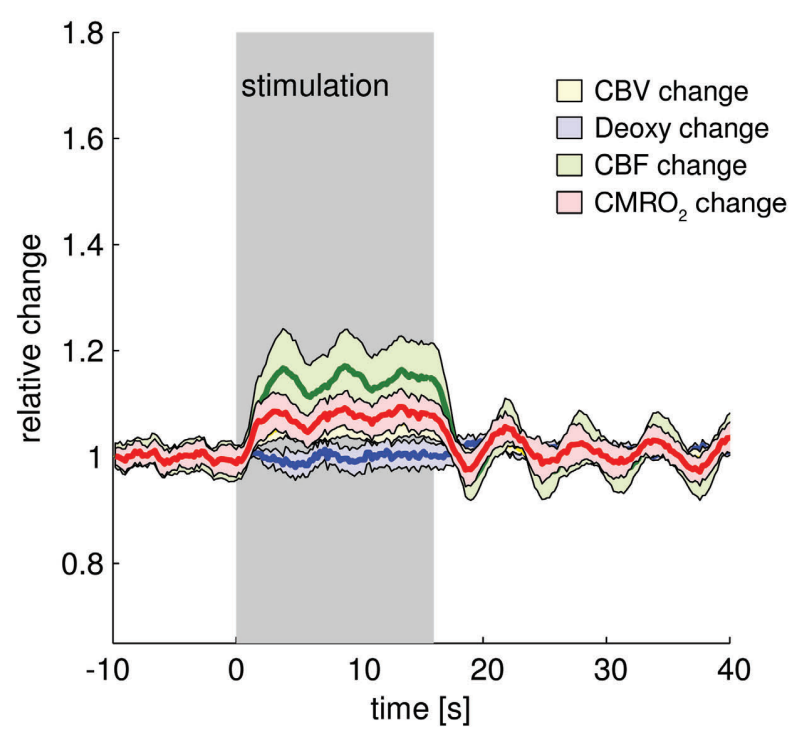

FIGURE 5 |When the CBF response is pharmacologically reduced, the deoxy-Hb response is abolished (from Leithner et al., 2010).

\section{rCBF response}

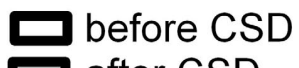
$\square$ after CSD

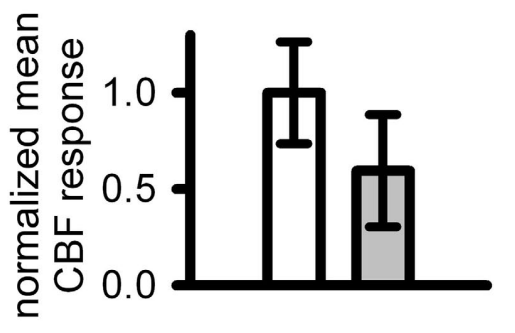

\section{rCBO response}

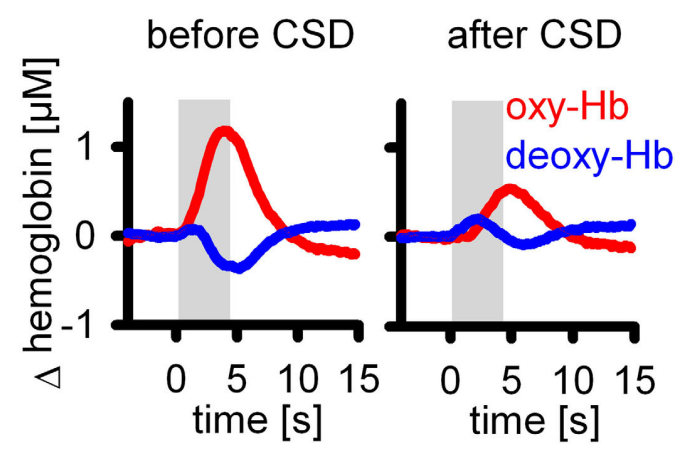

FIGURE 6 | Altered neurovascular coupling within the first hour following CSD in anesthetized rats.

CBF and oxygen metabolism may be altered under conditions of anesthesia, drug intake or disease (calibrated BOLD-fMRI). Only recently, it has been shown that under sevoflurane anesthesia, baseline $\mathrm{CBF}$ and the task-induced changes in CBF decreased, whereas a larger change in BOLD was observed (Qiu et al., 2008). While comparing functional activation induced BOLD responses in younger and older subjects, Ances et al. (2009) found a significantly smaller BOLD response for the older age group despite similar fractional $\mathrm{CBF}$ and $\mathrm{CMRO}_{2}$ changes and showed that the weaker BOLD response for the older is explained by a reduction in baseline CBF. In another study reduced activation of the frontal cortex has been shown in patients with bipolar disorders performing a cognitive task despite normal vascular responses to hypercapnia (calibrated fNIRS) (Matsuo et al., 2007). In addition, blood flow and blood oxygenation responses may not only be affected in their amplitude but also in their time course dynamics. For example, a study on stroke patients did show a longer time to peak in the BOLD activation time course, presumably reflecting a disturbed cerebral autoregulation (Altamura et al., 2009). Other fMRI studies on human subjects found a prolonged BOLD response in patients with hemodynamically significant stenoses (Roc et al., 2006) or a changed temporal dynamic of the BOLD response under caffeine (Liau et al., 2008). An fNIRS study on patients with cerebral microangiopathy reports a reduced amplitude of the hemodynamic response in tight correlation with behavioral deficits. In addition, an early deoxygenation of hemoglobin right after stimulation onset as well as a delay of the hemodynamic response is described, indicating not only frontal dysfunction but also an impairment of neurovascular coupling in cerebral microangiopathy (Schroeter et al., 2007). Functional MRIguided neurosurgery is another field of application for BOLD-fMRI 
as a non-invasive imaging technique. Despite its attractiveness for presurgical mapping to localize cerebral function in areas within or near tissue intended for neurosurgical resection (for review see Matthews et al., 2006), its reliability and thus applicability in brain tumor patients has been questioned (Fujiwara et al., 2004; Bartoš et al., 2009). In accordance with this critique, a very recent study using a multimodal approach in patients with primary brain tumors shows strong evidence for an impairment of vascular reactivity near meningiomas and high grade gliomas (Jiang et al., 2010). In addition, several studies on patients suffering from cerebrovascular diseases such as stroke or carotid artery stenosis explicitly used BOLD imaging to investigate impaired vascular function during preserved neuronal activity (Hamzei et al., 2003; Rossini et al., 2004; Krainik et al., 2005; Roc et al., 2006). In line with the above mentioned results from animal studies, these examples from human studies provide additional evidence that the deoxy-Hb and the BOLD response alone can result in a misleading interpretation of underlying physiological changes. Therefore they further support the need of knowledge on pathophysiological interferences and the use of controlled methods like calibrated BOLD-fMRI or ANIRS in situations where the normal coupling between $\mathrm{CBF}$ and oxygen metabolism in respect to amplitude and time course might be altered.

Despite the numerous studies with data from experimental and clinical studies in animals and humans that critically assess the reliability of vascular parameters to measure neuronal function under altered coupling conditions (Matthews et al., 2006; Iannetti and Wise, 2007; Wierenga and Bondi, 2007; Fleisher et al., 2009), the implementation of this knowledge in clinical studies in patients is still far from being established. Not only prior but also very recent studies on patients with ischemic stroke (Loubinoux et al., 2003; Enzinger et al., 2008), multiple sclerosis (Reddy et al., 2000; White et al., 2009), Parkinson's disease (Schonberg et al., 2010), Alzheimer's disease and dementia (Sauer et al., 2006; Thiyagesh et al., 2009) and schizophrenia (Huang et al., 2010) still do not control for impaired coupling, disturbed vascular reactivity or altered resting levels of blood volume, $\mathrm{CBF}, \mathrm{CBO}$, or metabolism as possible confounders to their findings. Therefore, some of these studies may bear the risk of generating a false negative (or false positive) conclusion. Further experimental and clinical studies will help us to identify any involvement of the vascular system, particularly in diseases putatively based on parenchymal alterations.

A number of suggestions for a general improvement of BOLDfMRI studies during possibly impaired neurovascular coupling have been published by Iannetti and Wise (2007). As more experimental evidence from experimental (animal) studies becomes available, anticipation of changes in amplitude or dynamics of BOLD, CBF,

\section{REFERENCES}

Alle, H., Roth, A., and Geiger, J. R.P. (2009). Energy-efficient action potentials in hippocampal mossy fibers. Science 325, 1405-1408.

Altamura, C., Reinhard, M., Vry, M. S., Kaller, C. P., Hamzei, F., Vernieri, F., Rossini, P. M., Hetzel, A., Weiller, C., and Saur, D. (2009). The longitudinal changes of BOLD response and cerebral hemodynamics from acute to subacute stroke. A fMRI and TCD study. BMC Neurosci. 10, 1-12.

Ances, B. M., Buerk, D. G., Greenberg, J. H., and Detre, J. A. (2001a). Temporal dynamics of the partial pressure of brain tissue oxygen during functional forepaw stimulation in rats. Neurosci. Lett. 306, 106-110.

Ances, B. M., Wilson, D. F., Greenberg, J. H., and Detre, J. A. (2001b). Dynamic changes in cerebral blood flow, $\mathrm{O}_{2}$
$\mathrm{CMRO}_{2}$, and CBV may be used to optimize imaging protocols. For example, when reduced CBF responses abolish BOLD responses, brain activity may still reliably be measured with CBF based fMRI methods, if reduced amplitudes are accounted for. CBF based neuroimaging also has the advantage of a higher spatial resolution (Duong et al., 2001) since it has been shown to enhance microcirculatory areas (Royl et al., 2006). In addition, it can provide quantitative values (Leithner et al., 2008). On the other hand, if neurovascular coupling is completely blocked and CBF responses are abolished, neuronal activity may lead to an increase in deoxy-Hb and a decrease of the BOLD-signal, which could again be used to detect active brain areas as long as reversed signal characteristics are taken into account. Results of such studies should always be interpreted very carefully. Maximal endeavor should be made to gather complementary data (electrophysiological, baseline physiological parameters, control tasks etc.) to at least partly control for the lack of validation studies linking neuronal activity with vascular parameters in such experiments. In addition to gathering more data from experimental studies, quality standards should be agreed on and established for the publication of functional activation studies performed in the environment of impaired neurovascular coupling.

\section{SUMMARY AND OUTLOOK}

Functional MRI may not be reliable in pathological states, as neuronal activity may be preserved but deoxy-Hb changes absent. Alterations in $\mathrm{rCBF}$ or arterial blood oxygenation, hyper- or hypocapnia, changes in intracranial pressure, severe decreases of systemic blood pressure, cortical spreading depressions, medication or brain or vascular diseases may alter the relationship between neuronal activity and BOLD-signals, with the risk of false negative findings in brain activation studies. To guarantee correct interpretation of these studies, future research needs to deepen our understanding of the basic mechanisms of neurovascular coupling and the specific characteristics of disturbed neurovascular coupling in the diseased brain.

\section{AUTHORIZATION FOR THE USE OF EXPERIMENTAL ANIMALS}

All animal experiments were performed in accordance with institutional and national guidelines and regulations and were approved by the "Landesamt für Arbeitsschutz, Gesundheitsschutz und technische Sicherheit Berlin."

\section{ACKNOWLEDGMENT}

This work was supported by the Deutsche Forschungsgemeinschaft, the Hermann and Lilly Schilling Foundation and the European Union (EFRE). We thank Dr. Jörn Gethmann and Marco Foddis for excellent technical assistance. tension, and calculated cerebral metabolic rate of $\mathrm{O}_{2}$ during functional activation using oxygen phosphorescence quenching. J. Cereb. Blood Flow Metab. 21, 511-516.

Ances, B. M., Liang, C. L., Leontiev, O., Perthen, J.E., Fleisher, A. S., Lansing, A. E., and Buxton, R. B. (2009). Effects of aging on cerebral blood flow, oxygen metabolism, and blood oxygenation level dependent responses to visual stimulation. Hum. Brain Mapp. 30, 1120-1132.

Auer, D. P. (2008). Spontaneous lowfrequency blood oxygenation leveldependent fluctuations and functional connectivity analysis of the 'resting' brain. Magn. Reson. Imaging 26, 1055-1064.

Bartoš, R., Jech, R., Vymazal, J., Petrovický, P., Vachata, P., Hejčl, A., Zolal, A., and Sameš, M. (2009). Validity of primary 
motor area localization with fMRI versus electric cortical stimulation: a comparative study. Acta Neurochir. 151, 1071-1080.

Bruhn, H., Fransson, P., and Frahm, J. (2001). Modulation of cerebralblood oxygenation by indomethacin: MRI at rest and functional brain activation. J. Magn. Reson. Imaging 13, 325-334.

Buxton, R. B., Uludag, K., Dubowitz, D. J., and Liu, T. T. (2004). Modeling the hemodynamic response to brain activation. Neuroimage 23, S220-S233.

Chen, J. J., and Pike, G. B. (2010). Global cerebral oxidative metabolism during hypercapnia and hypocapnia in humans: implications for BOLD fMRI. J. Cereb. Blood Flow Metab. 30, 1094-1099.

Chen, Y., and Parrish, T. B. (2009). Caffeine dose effect on activationinduced BOLD and CBF responses. Neuroimage 46, 577-583.

Davis, T. L., Kwong, K. K., Weisskoff, R. M., and Rosen, B. R. (1998). Calibrated functional MRI: mapping the dynamics of oxidative metabolism. Proc. Natl. Acad. Sci. U.S.A. 95, 1834-1839.

Dunn, A. K., Devor, A., Dale, A. M., and Boas, D. A. (2005). Spatial extent of oxygen metabolism and hemodynamic changes during functional activation of the rat somatosensory cortex. Neuroimage 27, 279-290.

Duong, T. Q., Kim, D. S., Ugurbil, K., and Kim, S. G. (2001). Localized cerebral blood flow response at submillimeter columnar resolution. Proc. Natl. Acad. Sci. U.S.A. 98, 10904-10909.

Enager, P., Piilgaard, H., Offenhauser, N., Kocharyan, A., Fernandes, P., Hamel, E., and Lauritzen, M. (2009). Pathway-specific variations in neurovascular and neurometabolic coupling in rat primary somatosensory cortex. J. Cereb. Blood Flow Metab. 29, 976-986.

Enzinger, C., Johansen-Berg, H., Dawes, H., Bogdanovic, M., Collett, J., Guy, C., Ropele, S., Kischka, U., Wade, D., Fazekas, F., and Matthews, P. M. (2008). Functional MRI correlates of lower limb function in stroke victims with gait impairment. Stroke 39, 1507-1513.

Fleisher, A. S., Podraza, K. M., Bangen, K. J., Taylor, C., Sherzai, A., Sidhar, K., Liu, T. T., Dale, A. M., and Buxton, R. B. (2009). Cerebral perfusion and oxygenation differences in Alzheimer's disease risk. Neurobiol. Aging 30, 1737-1748.

Fox, P. T., Raichle, M. E., Mintun, M. A., and Dence, C. (1988). Nonoxidative glucose consumption during focal physiologic neural activity. Science 241, 462-464.
Füchtemeier,M.,Leithner,C.,Offenhauser, N., Foddis, M., Kohl-Bareis, M., Dirnagl, U., Lindauer, U., and Royl, G. (2010). Elevating intracranial pressure reverses the decrease in deoxygenated hemoglobin and abolishes the poststimulus overshoot upon somatosensory activation in rats. Neuroimage 52, 445-454.

Fujiwara, N., Sakatani, K., Katayama, Y., Murata, Y., Hoshino, T., Fukaya, C., and Yamamoto, T. (2004). Evokedcerebral blood oxygenation changes in false-negative activations in BOLD contrast functional MRI of patients with brain tumors. Neuroimage 21, 1464-1471.

Geocadin, R. G., Koenig, M. A., Stevens, R. D., and Peberdy, M. A. (2006). Intensive care for brain injury after cardiac arrest: therapeutic hypothermia and related neuroprotective strategies. Crit. Care Clin. 22, 619-636.

Gjedde, A., Marrett, S., and Vafaee, M. (2002). Oxidative and nonoxidative metabolism of excited neurons and astrocytes. J. Cereb. Blood Flow Metab. $22,1-14$.

Hamzei, F., Knab, R., Weiller, C., and Röther, J. (2003). The influence of extra- and intracranial artery disease on the BOLD signal in FMRI. Neuroimage 20, 1393-1399.

He, B. J., and Raichle, M. E. (2009). The fMRI signal, slow cortical potential and consciousness. Trends Cogn. Sci. 13, 302-309.

Huang, X. -Q., Lui, S., Deng, W., Chan, R. C. K., Wu, Q. -Z., Jiang, L. -J., Zhang, J. -R., Jia, Z. -Y., Li, X. -L., Li, F., Chen, L., Li, T., and Gong, Q. -Y. (2010). Localization of cerebral functional deficits in treatment-naive, first-episode schizophrenia using resting-state fMRI. Neuroimage 49, 2901-2906.

Huppert, T. J., Jones, P. B., Devor, A., Dunn, A. K., Teng, I. C., Dale, A. M., and Boas, D. A. (2009). Sensitivity of neural-hemodynamic coupling to alterations in cerebral blood flow during hypercapnia. J. Biomed. Opt. 14, 044038.

Iadecola, C. (2004). Neurovascular regulation in the normal brain and in Alzheimer's disease. Nat. Rev. Neurosci. 5, 347-360.

Iannetti, G. D., and Wise, R. G. (2007). BOLD functional MRI in disease and pharmacological studies: room for improvement? Magn. Reson. Imaging 25, 978-988.

Jiang, Z., Krainik, A., David, O., Salon, C., Troprès, I., Hoffmann, D., Pannetier, N., Barbier, E. L., Bombìn, E. R., Warnking, J., Pasteris, C., Chabardes, S., Berger, F., Grand, S., Segebarth, C., Gay, E., and Le Bas, J. -F. (2010). Impaired fMRI acti- vation in patients with primary brain tumors. Neuroimage 52, 538-548.

Jones, M., Berwick, J., Hewson-Stoate, N., Gias, C., and Mayhew, J. (2005). The effect of hypercapnia on the neural and hemodynamic responses to somatosensory stimulation. Neuroimage 27, 609-623.

Kennerley, A. J., Berwick, J., Martindale, J., Johnston, D., Zheng, Y., and Mayhew, J. E. (2009). Refinement of optical imaging spectroscopy algorithms using concurrent BOLD and CBV fMRI. Neuroimage 47, 1608-1619.

Kety, S. S., Shenkin, H. A., and Schmidt, C. F. (1948). The effects of increased intracranial pressure on cerebral circulatory functions in man. J. Clin. Invest. 27, 493-499.

Krainik, A., Hund-Georgiadis, M., Zysset, S., and von Cramon, D. Y. (2005). Regional impairment of cerebrovascular reactivity and BOLD signal in adults after stroke. Stroke 36, 1146-1152.

Leithner, C., Gertz, K., Schröck, H., Priller, J., Prass, K., Steinbrink, J., Villringer, A., Endres, M., Lindauer, U., Dirnagl, U., and Royl, G. (2008). A flow sensitive alternating inversion recovery (FAIR)-MRI protocol to measure hemispheric cerebral blood flow in a mouse stroke model. Exp. Neurol. 210, 118-127.

Leithner, C., Royl, G., Offenhauser, N., Füchtemeier, M., Kohl-Bareis, M., Villringer, A., Dirnagl, U., and Lindauer, U. (2010). Pharmacological uncoupling of activation induced increases in $\mathrm{CBF}$ and $\mathrm{CMRO}_{2}$.J. Cereb. Blood Flow Metab. 30, 311-322.

Leontiev, O., and Buxton, R. B. (2007). Reproducibility of BOLD, perfusion, and $\mathrm{CMRO}_{2}$ measurements with calibrated-BOLD fMRI. Neuroimage 35, 175-184.

Liau, J., Perthen, J. E., and Liu, T. T. (2008). Caffeine reduces the activation extent and contrast-to-noise ratio of the functional cerebral blood flow response but not the BOLD response. Neuroimage 42, 296-305.

Lin, A. -L., Fox, P. T., Yang, Y., Lu, H., Tan, L. -H., and Gao, J. -H. (2008). Evaluation of MRI models in the measurement of $\mathrm{CMRO}_{2}$ and its relationship with CBF. Magn. Reson. Med. 60, 380-389.

Lindauer, U., Gethmann, J., Kühl, M., Kohl-Bareis, M., and Dirnagl, U. (2003). Neuronal activity-induced changes of local cerebral microvascular blood oxygenation in the rat: effect of systemic hyperoxia or hypoxia. Brain Res. 975, 135-140.

Lindauer, U., Leithner, C., Kaasch, H., Rohrer, B., Foddis, M., Füchtemeier, M., Offenhauser, N., Steinbrink, J., Royl, G., Kohl-Bareis, M., and Dirnagl,
U. (2010). Neurovascular coupling in rat brain operates independent of hemoglobin deoxygenation. J. Cereb. Blood Flow Metab. 30, 757-768.

Lindauer, U., Royl, G., Leithner, C., Kühl, M., Gold, L., Gethmann, J., Kohl-Bareis, M., Villringer, A., and Dirnagl, U. (2001). No evidence for early decrease in blood oxygenation in rat whisker cortex in response to functional activation. Neuroimage 13, 988-1001.

Logothetis, N. K. (2008). What we can do and what we cannot do with fMRI. Nature 453, 869-878.

Logothetis, N. K., Pauls, J., Augath, M., Trinath, T., and Oeltermann,A. (2001). Neurophysiological investigation of the basis of the fMRI signal. Nature 412, 150-157.

Loubinoux, I., Carel, C., Pariente, J., Dechaumont, S., Albucher, J. -F., Marque, P., Manelfe, C., and Chollet, F. (2003). Correlation between cerebral reorganization and motor recovery after subcortical infarcts. Neuroimage 20, 2166-2180.

Lu, C. -M., Zhang, Y. -J., Biswal, B. B., Zang, Y. -F., Peng, D. -L., and Zhu, C. -Z. (2010). Use of fNIRS to assess resting state functional connectivity. $J$. Neurosci. Methods 186, 242-249.

Masamoto, K., Vazquez, A., Wang, P., and Kim, S. -G. (2008). Trial-by-trial relationship between neural activity, oxygen consumption, and blood flow responses. Neuroimage 40, 442-450.

Mathiesen,C., Caesar,K.,Akgoren, N., and Lauritzen, M. (1998). Modification of activity-dependent increases of cerebral blood flow by excitatory synaptic activity and spikes in rat cerebellar cortex. J. Physiol. (Lond.) 512, 555-566.

Mathiesen, C., Caesar, K., and Lauritzen, M. (2000). Temporal coupling between neuronal activity and blood flow in rat cerebellar cortex as indicated by field potential analysis. J. Physiol. 523( $\mathrm{Pt}$ 1), 235-246.

Matsuo, K., Kouno, T., Hatch, J. P., Seino, K., Ohtani, T., Kato, N., and Kato, T. (2007). A near-infrared spectroscopy study of prefrontal cortex activation during a verbal fluency task and carbon dioxide inhalation in individuals with bipolar disorder. Bipolar Disord. 9, 876-883.

Matthews, P. M., Honey, G. D., and Bullmore, E. T. (2006). Applications of fMRI in translational medicine and clinical practice. Nat. Rev. Neurosci. 7, 732-744.

Monti, M. M., Vanhaudenhuyse, A., Coleman, M. R., Boly, M., Pickard, J. D., Tshibanda, L., Owen, A. M., and Laureys, S. (2010). Willful modulation of brain activity in disorders of 
consciousness. N. Engl. J. Med. 362, 579-589.

Nagaoka, T., Zhao, F., Wang, P., Harel, N., Kennan, R. P., Ogawa, S., and Kim, S. -G. (2006). Increases in oxygen consumption without cerebral blood volume change during visual stimulation under hypotension condition. J. Cereb. Blood Flow Metab. 26, 1043-1051.

Obrig, H., Heekeren, H., Ruben, J., Wenzel, R., Ndayisaba, J. P., Dirnagl, U., and Villringer, A. (1996). Continuous spectrum near-infrared spectroscopy approach in functional activation studies in the human adult. Proc SPIE 2926, 58-66.

Obrig, H., and Villringer, A. (2003). Beyond the visible - imaging the human brain with light. J. Cereb. Blood Flow Metab. 23, 1-18.

Offenhauser, N., Royl, G., Leithner, C., Dirnagl, U., and Lindauer, U. (2007). Sustained alteration of neurometabolic, vascular coupling by cortical spreading depression in rat somatosensory cortex. J. Cereb. Blood Flow Metab. 27(Suppl. 1), BO13-07.

Offenhauser, N., Thomsen, K., Caesar, K., and Lauritzen, M. (2005). Activityinduced tissue oxygenation changes in rat cerebellar cortex: interplay of postsynaptic activation and blood flow. J. Physiol. 565, 279-294.

Piilgaard, H., and Lauritzen, M. (2009). Persistent increase in oxygen consumption and impaired neurovascular coupling after spreading depression in rat neocortex. J. Cereb. Blood Flow Metab. 29, 1517-1527.

Qiu, M., Ramani, R., Swetye, M., Rajeevan, N., and Constable, R. T. (2008). Anesthetic effects on regional CBF, BOLD, and the coupling between taskinduced changes in CBF and BOLD: an fMRI study in normal human subjects. Magn. Reson. Med. 60, 987-996.

Rack-Gomer, A. L., Liau, J., and Liu, T. T. (2009). Caffeine reduces resting-state BOLD functional connectivity in the motor cortex. Neuroimage 46, 56-63.
Rauch, A., Rainer, G., and Logothetis, N. K. (2008). The effect of a serotonininduced dissociation between spiking and perisynaptic activity on BOLD functional MRI. Proc. Natl. Acad. Sci. U.S.A. 105, 6759-6764.

Reddy, H., Narayanan, S., Arnoutelis, R., Jenkinson, M., Antel, J., Matthews, P. M., and Arnold, D. L. (2000). Evidence for adaptive functional changes in the cerebral cortex with axonal injury from multiple sclerosis. Brain 123, 2314-2320.

Roc, A. C., Wang, J., Ances, B. M., Liebeskind, D. S., Kasner, S. E., and Detre, J. A. (2006). Altered hemodynamics and regional cerebral blood flow in patients with hemodynamically significant stenoses. Stroke 37 , 382-387.

Rossini, P. M., Altamura, C., Ferretti, A., Vernieri, F., Zappasodi, F., Caulo, M., Pizzella, V., Del Gratta, C., Romani, G. -L., and Tecchio, F. (2004). Does cerebrovascular disease affect the coupling between neuronal activity and local haemodynamics? Brain 127, 99-110.

Roy, C., and Sherrington, C. (1890). On the regulation of the blood supply of the brain. J. Physiol. 11, 85-108.

Royl, G., Füchtemeier, M., Leithner, C., Megow,D.,Offenhauser,N.,Steinbrink, J., Kohl-Bareis, M., Dirnagl, U., and Lindauer, U. (2008). Hypothermia effects on neurovascular coupling and cerebral metabolic rate of oxygen. Neuroimage 40, 1523-1532.

Royl, G., Leithner, C., Sellien, H., Müller, J. P., Megow, D., Offenhauser, N., Steinbrink, J., Kohl-Bareis, M., Dirnagl, U., and Lindauer, U. (2006). Functional imaging with Laser Speckle Contrast Analysis: vascular compartment analysis and correlation with Laser Doppler Flowmetry and somatosensory evoked potentials. Brain Res. 1121, 95-103.

Sacco, R. L., Chong, J. Y., Prabhakaran, S., and Elkind, M. S. V. (2007).
Experimental treatments for acute ischaemic stroke. Lancet 369, 331-341.

Sauer, J., ffytche, D. H., Ballard, C., Brown, R. G., and Howard, R. (2006). Differences between Alzheimer's disease and dementia with Lewy bodies: an fMRI study of task-related brain activity. Brain 129, 1780-1788.

Schonberg, T., O’Doherty, J. P., Joel, D., Inzelberg, R., Segev, Y., and Daw, N. D. (2010). Selective impairment of prediction error signaling in human dorsolateral but not ventral striatum in Parkinson's disease patients: evidence from a model-based fMRI study. Neuroimage 49, 772-781.

Schreiber, A., Hubbe, U., Ziyeh, S., and Hennig, J. (2000). The influence of gliomas and nonglial space-occupying lesions on blood-oxygen-leveldependent contrastenhancement.AJNR Am. J. Neuroradiol. 21, 1055-1063.

Schroeter, M. L., Cutini, S., Wahl, M. M. Scheid, R., and Yves von Cramon, D. (2007). Neurovascular coupling is impaired in cerebral microangiopathy - an event-related Stroop study. Neuroimage 34, 26-34.

St Lawrence, K. S., Ye, F. Q., Lewis, B. K., Frank, J. A., and McLaughlin, A. C. (2003). Measuring the effects of indomethacin on changes in cerebral oxidative metabolism and cerebral blood flow during sensorimotor activation. Magn. Reson. Med. 50, 99-106.

Thiyagesh, S. N., Farrow, T. F. D., Parks, R. W., Accosta-Mesa, H., Young, C., Wilkinson, I. D., Hunter, M. D., and Woodruff, P. W. R. (2009). The neural basis of visuospatial perception in Alzheimer's disease and healthy elderly comparison subjects: an fMRI study. Psychiatry Res. 172, 109-116.

Thompson, J. K., Peterson, M. R., and Freeman, R. D. (2003). Single-neuron activity and tissue oxygenation in the cerebral cortex. Science 299, 1070-1072.
Uludag, K., Müller-Bierl, B., and Ugurbil, K. (2009). An integrative model for neuronal activity-induced signal changes for gradient and spin echo functional imaging. Neuroimage 48, 150-165.

White, A., Lee, J., Light, A., and Light, K. (2009). Brain activation in multiple sclerosis: a BOLD fMRI study of the effects of fatiguing hand exercise. Mult. Scler. 15, 580-586.

Wierenga, C., and Bondi, M. (2007). Use of functional magnetic resonance imaging in the early identification of Alzheimer's disease. Neuropsychol. Rev. 17, 127-143.

Zappe, A. C., Uludag, K., Oeltermann, A., Ugurbil, K., and Logothetis, N. K. (2008). The influence of moderate hypercapnia on neural activity in the anesthetized nonhuman primate. Cereb. Cortex 18, 2666-2673.

Conflict of Interest Statement: The authors declare that the research was conducted in the absence of any commercial or financial relationships that could be construed as a potential conflict of interest.

Received: 22 February 2010; paper pending published: 26 April 2010; accepted: 20 July 2010; published online: 04 October 2010. Citation: Lindauer $U$, Dirnagl $U$, Füchtemeier M, Böttiger C, Offenhauser $N$, Leithner C and Royl G (2010) Pathophysiological interference with neurovascular coupling - when imaging based on hemoglobin might go blind. Front. Neuroenerg. 2:25. doi: 10.3389/ fnene.2010.00025

Copyright (c) 2010 Lindauer, Dirnagl, Füchtemeier, Böttiger, Offenhauser, Leithner and Royl. This is an open-access article subject to an exclusive license agreement between the authors and the Frontiers Research Foundation, which permits unrestricted use, distribution, and reproduction in any medium, provided the original authors and source are credited. 